\title{
Formation and internal structure of superdense dark matter clumps and ultracompact minihaloes
}

\author{
V.S. Berezinsky, , 因 V.I. Dokuchaev, ${ }^{2}$ 团 and Yu.N. Eroshenko, ${ }^{2}$ 团 \\ ${ }^{1}$ INFN, Laboratori Nazionali del Gran Sasso, Center for Astroparticle Physics at LNGS (CFA), \\ I-67010 Assergi (AQ), Italy \\ ${ }^{2}$ Institute for Nuclear Research of the Russian Academy of Sciences \\ 60th October Anniversary Prospect 7a, 117312 Moscow, Russia
}

(Dated: August 19, 2018)

\begin{abstract}
We discuss the formation mechanisms and structure of the superdense dark matter clumps (SDMC) and ultracompact minihaloes (UCMH), outlining the differences between these types of DM objects. We define as SDMC the gravitationally bounded DM objects which have come into virial equilibrium at the radiation-dominated (RD) stage of the universe evolution. Such objects can be formed from the isocurvature (entropy) density perturbations or from the peaks in the spectrum of curvature (adiabatic) perturbation. The axion miniclusters (Kolb and Tkachev 1994) are the example of the former model. The system of central compact mass (e. g. in the form of SDMC or primordial black hole $(\mathrm{PBH})$ ) with the outer DM envelope formed in the process of secondary accretion we refer to as UCMH. Therefore, the SDMC can serve as the seed for the UCMH in some scenarios. Recently, the SDMC and UCMH were considered in the many works, and we try to systematize them here. We consider also the effect of asphericity of the initial density perturbation in the gravitational evolution, which decreases the SDMC amount and, as the result, suppresses the gamma-ray signal from DM annihilation.
\end{abstract}

\section{INTRODUCTION}

Although the existence of DM is reliably established, the nature of the DM particles is still unknown. There are several well motivated DM models, which allow the direct and indirect experimental verification in the near future. The most bright indirect signature is the particles annihilation, and this process could be boosted inside the dense DM clumps.

In the standard scenario the DM clumps are formed from the CMB-normalized power-law spectrum of perturbations. The clumps participate in the hierarchical clustering, and finally have near power-law mass spectrum, obtained both in the numerical simulations [1] and analytically [2]. In the case of neutralino DM the minimum mass of the clumps is $M_{\min } \sim 10^{-6} M_{\odot}$ (see e. g. [3], [4]). These lightest clumps are formed at the red-shift $z \sim 50$. The situation could be very different for other DM particle candidates, e. g. in the case of the superheavy DM particles [5], and/or for the non-standard spectrum of perturbations. DM clumps can be formed very early in the beginning of the matter-dominated or even at the RD-stage from the large density perturbations of different nature: peaks in the spectrum of perturbations generated during inflation or at phase transitions in the early universe, or with the PBHs or cosmic string loops as the seeds. In this paper we consider in some details the two somehow related types of DM clumps: SDMC and UCMH.

\footnotetext{
* e-mail: berezinsky@lngs.infn.it

$\dagger$ e-mail: dokuchaev@lngs.infn.it

$\ddagger$ e-mail: eroshenko@inr.ac.ru
}

In 6] the formation of SDMCs at the RD-stage evolution of the universe from the entropy perturbations (disturbances of matter content with constant curvature) was considered. The 7] generalized this formalism for the adiabatic perturbations, and it was found that the $\mathrm{PBHs}$ and SDMCs can be connected due to their common origin from the same spectrum of density perturbations. SDMC forms if the perturbation is not sufficient for formation of PBH. Such SDMCs can be figuratively called as "failed black holes", because in the case of higher perturbation the PBH would be formed instead of SDMC. A similar idea was discussed in [8], [9], [10], [1], [12], [13], and in more detail - in [14]. It is important that the observational cosmic gamma-rays constraints can limit the DM annihilation and the spectrum of initial perturbations [10], 14], 15]. However, to establish reliably such restrictions, it is necessary to determine the structure of SDMC, especially in its central region.

Later, at the matter-dominated stage, the DM concentrates around the SDMCs in the process of secondary accretion [16] forming the halo with density profile $\propto r^{-9 / 4}$ 16], 6]. The similar halos can grow around PBHs [17], [18], 19], 20], 21] 22]. The DM clumps, formed on the dust-like stage, probably have the density profiles of the Gurevich-Zybin type $r^{-1.8}$ 23], 24], 25], if these objects are isolated. Gurevich-Zybin theory explains the formation of singular density profile from the initially smooth perturbation. The Navarro-Frenk-White (NFW) profile is applicable, if the objects were not isolated during their formation but experienced hierarchical clustering. Such a transition from NFW to a steeper inner profile near the minimum mass scale (objects at this scale are formed almost as isolated) was observed in the work [26]. Gurevich-Zybin profile should give smaller annihilation signal compared with the calculations [14], where the 
$r^{-9 / 4}$ profile was used. The simulations of 27$]$ indicate in favor of the $r^{-9 / 4}$ Fillmore \& Goldreich / Bertschinger secondary infall profile even for isolated halos forming without a compact central object. Therefore more work is needed to clarify this topic.

If the UCMH formed around a superdense SDMC, which virialised at the RD stage, the central density is determined by the SDMC's structure. First, the calculation of the average density of SDMC requires the formalism of [6], 7], [5]. Second, the Gurevich-Zybin or steeper profile probably is formed in the SDMCs because of their isolation. Third, the question about the radius of the central core has not yet definite solution. Really, the central density of halos and subhalos is one of the unresolved problem in the observations and in the theory of the hierarchical DM structure formation.

Using the conventional models of WIMPs annihilation, in the papers 10], 12], 13], [14] it was concluded from the absence of the observed annihilation signal, that the SDMCs can constitute only $\ll 1$ fraction of DM. A similar result was obtained in [11] from the influence of the early annihilation on recombination and taking into account the known limitations to this effect from the CMB observations. The influence of UCMHs on the intergalactic medium and reionisation of the universe was studied in details by [21]. The restrictions on the UCMHs in the decaying dark mater model were considered in 28]. Some restrictions were also obtained from the upper limits on the neutrino signals from annihilation [29]. The lack of the observed gamma-ray point sources demonstrates that in the epoch of $e^{+} e^{-}$annihilation the value of perturbations at the horizon crossing was less than $10^{-3}[8]$. The SDMC, if they exists, were not destroyed in the tidal interactions during the formation of structures at large scales. One may propose the non-sphericity of the density perturbations as the effects that could suppress the formation and diminish the amount of the SDMC. In this paper we will show that non-sphericity plays the important role, and the SDMC number can be suppressed by several orders of magnitudde. In the papers [8], 10], 11], [22], 14], 12], 13] the maximum density in the center of the UCMH is found from the effect of the particles annihilation. It was assumed that since the formation moment of UCMH the more and more particles have time to annihilate, the region of the annihilation expands, and therefore the radius of the core increases. This approach goes back to [30]. In the opposite approach 31], 32], proposed earlier, it was taken into account that in addition to the annihilation the continuous stream of particles into the center of the UCMH exists, so that the core radius does not change significantly over time.

In addition to the annihilation there are other restrictions on the central density and radius of the core. In [14] the core radius $R_{c}$ was estimated by considering the transverse velocities which resulted in $R_{c} / R \sim 3 \times 10^{-7}$, where $R$ is the virial radius. Restrictions on the phase space density come from the Liouville theorem, from the tidal forces 33], from the presence of substructures and the other sources of entropy generation [34]. It is likely that the clumps have the core radius with $10^{-3}-10^{-2}$ fraction of $R$ as it follows from the numerical simulations of [1] (Fig. 2).

The important question is the final mass of the growing UCMH. In the papers [19], [8], [11], 22], [12], 13] it was assumed that the ceasing of the mass growth during the secondary accretion occurred at $z \sim 10-30$, when the large-scale structure began forming actively. Previously, a rigorous criterion for the end of the growth was found in the papers [17], [18] and it also will be discussed in this paper.

\section{FORMATION OF SUPERDENSE CLUMPS}

For the clumps to be formed at the RD-stage of the universe evolution, the spectrum of perturbations must have an excess at small scale. The ordinary CMBnormalised power-low spectrum with the observed power index $n_{s} \simeq 0.96$ provides too small perturbations at the RD-stage for the clumps formation there. Really, the spectrum of the curvature perturbations in this case is 35]

$$
\mathcal{P}_{\mathcal{R}}=A_{\mathcal{R}}\left(\frac{k}{k_{*}}\right)^{n_{s}-1}
$$

where $k_{*} / a_{0}=0.002 \mathrm{Mpc}^{-1}, A_{\mathcal{R}}=(2.46 \pm 0.09) \times 10^{-9}$, and $n_{s}=0.960 \pm 0.014$. Therefore, the typical amplitude of the perturbations $\Delta_{\mathcal{R}} \simeq 5 \times 10^{-5}$ is insufficient for the clumps formation at the RD-stage.

For the further examples we consider the peak-like excess at some scale superimposed on the ordinary power low spectrum (11) of adiabatic perturbations. In the case of the sufficiently high peak, the clumps can be formed even at the RD-stage. The constraints on the possible parameters of the peak, its position at CDM mass scale $M$ and the peak's high $\delta_{H}$, are imposed by the $\mathrm{PBH}$ overproduction limits.

\section{A. Spherical collapse}

Let us outline formalism of the SDMCs formation at the RD-stage, which was developed in [6] for entropy perturbations and generalized for the curvature perturbations in [7]. We will start from the linear stage of evolution of the density perturbations. We will use the following notation: $x=k \eta$, where $k$ is the co-moving wave vector of the perturbation, which could be expressed through DM mass $M$ of the SDMC and $\eta$ is the conformal time with $d \eta=c d t / a(t)$. The adiabatic DM perturbation at $x \gg 1$ follows the law 35]

$$
\delta=-9 \Phi_{i}\left[\ln \left(\frac{x}{\sqrt{3}}\right)+\mathbf{C}-\frac{1}{2}\right]
$$


where $\Phi_{i}$ is the initial gravitational potential in the conformal Newtonian frame, which is related to the perturbation of curvature and to the perturbation of the radiation density at the horizon crossing as $\Phi_{i}=-2 \mathcal{R} / 3 \approx$ $-0.2 \delta_{H}$, and $\mathbf{C} \simeq 0.577$ is the Euler constant. The (2) serves as the initial condition for the evolution of DM density perturbation at the linear stage, then $\delta \ll 1$, according to the equation 35]

$$
y(y+1) \delta^{\prime \prime}+\left(1+\frac{3}{2} y\right) \delta^{\prime}-\frac{3}{2} \delta=0,
$$

where $y=a(\eta) / a_{\mathrm{eq}}$, and the prime denotes the derivative over $y$. This linear stage gives the initial conditions for the further nonlinear evolution.

Suppose that there is a positive density perturbation $\delta(\vec{r})$. The origin of coordinates $\vec{r}=0$ is chosen near the center of mass of the protohalo. In the first approximation the perturbation can be regarded as the spherically symmetric object. Denote by $M$ the DM mass within some spherical layer. The contribution of the pressure of the homogeneous relativistic component into the energymomentum tensor can be taken into account by replacing $\rho \rightarrow \rho+3 p c^{2}$. Then, the evolution of a spherical layer at the sub-horizon scales $r \ll c t$ obeys the equation

$$
\frac{d^{2} r}{d t^{2}}=-\frac{G\left(M_{h}+M\right)}{r^{2}}-\frac{8 \pi G \rho_{r} r}{3}+\frac{8 \pi G \rho_{\Lambda} r}{3}
$$

It is taken into account the possibility of a bare mass $M_{h}$, such as the mass of the black hole at the center of the layer. One may use the following parametrization:

$$
r=a(y) b(y) \xi
$$

where $\xi$ is the comoving coordinate. Then $b$ obeys the equation [6]

$$
y(y+1) b^{\prime \prime}+\left(1+\frac{3}{2} y\right) b^{\prime}+\frac{1}{2}\left(\frac{1+\delta_{i}}{b^{2}}-b\right)=0 .
$$

The Eq.(6) was solved in [6] for isocurvature (entropic) perturbations. In this case the initial velocity $d b / d y \simeq 0$, and the perturbation grows due to the initial value of the perturbation $\delta_{i} \neq 0$. According to [6] the central density of the clump is $140 \rho_{\mathrm{eq}} \delta_{i}^{3}\left(1+\delta_{i}\right)$, although our calculations gives for the mean density the smaller value $\simeq 17 \rho_{\text {eq }} \delta_{i}^{4}$.

For the adiabatic perturbations the nonzero initial quantity $d b / d y$ is specified according to the solution (2). To link the Eulerian and Lagrangian approaches, we put $b_{i}=\left(1+\delta_{i}\right)^{-1 / 3}$ at some initial $y_{i}$. For $x \gg 1$ and $y \ll 1$, we have the connection [7]

$$
x=\frac{\pi}{2^{2 / 3}}\left(\frac{3}{2 \pi}\right)^{1 / 6} \frac{y c}{M^{1 / 3} G^{1 / 2} \rho_{\mathrm{eq}}^{1 / 6}},
$$

then

$$
\left.b^{\prime}\right|_{y_{i}}=-\frac{\delta_{H} b_{i}^{4}}{2 y_{i} \phi}
$$

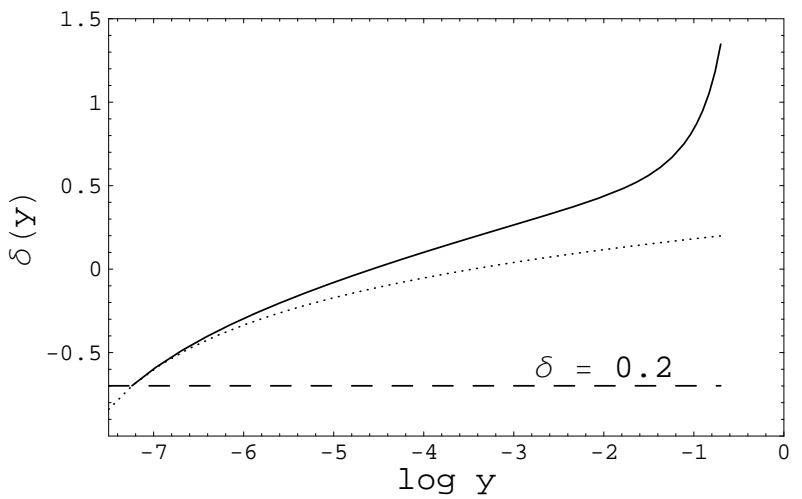

Figure 1. Example of the SDMC evolution with $M=$ $10^{-6} M_{\odot}$ and $\delta_{H}=0.05$. The solid curve was obtained by the numerical solution of (6) with the initial conditions according to (2), matched at $\delta=0.2$ (dashed curve). The dotted curve shows the extrapolation of the linear law (2) to the large times.

where $\phi \approx 0.817$. The Eq. (6) can be solved numerically. The moment $y_{i}$ is chosen in the region where (2) and (6) are both valid. By fixing $\delta_{i}=0.2$, we obtain $x_{i}$ and $y_{i}$ from the equations (2) and (7). The particular example of the $\delta=b^{-3}-1$ evolution is illustrated at Fig 1 .

The expansion of SDMC stops when $d r / d t=0$, which is equivalent to $d b / d y=-b / y[6]$. Let us use the index "max" for the quantities at the the moment of the stop, then the density and radius of the SDMC at this time are

$$
\rho_{\max }=\rho_{\mathrm{eq}} y_{\max }^{-3} b_{\max }^{-3}, \quad R_{\max }=\left(\frac{3 M}{4 \pi \rho_{\max }}\right)^{1 / 3}
$$

After virialization the final radius $R=R_{\max } / 2$ and therefore $\bar{\rho}=8 \rho_{\max }$. Virialization is the DM mixing inside the forming halo till the equilibrium state. It works due to the presence of radial osculations, large irregularities and non-spherical movements. In the terminology of LyndenBell [36], these processes are called "violent relaxation". The hierarchical clustering and associated relaxation also produce the universal density profiles, such as NavarroFrenk-White profile [37]. The new feature of the vitialization at the RD-stage is the disintegration of the very irregular protohalo. Indeed, if the protohalo is sufficiently elongated then the mass of the radiation inside the enveloping sphere will exceed the mass of DM, and the self-gravitation of the object will not hold the protohalo's parts together. The protohalo will decay and the parts of it will fly away one after another. To obtain the boundary of this regime of evolution, leading to the decay, the numerical simulations of SDMC at the RD-stage are desirable.

The SDMCs are formed due to the beginning of the matter dominated stages inside the local areas of the space, while on the average the universe is still at the RD-stage. The calculated $\bar{\rho}$ is shown at the Fig 2 . 


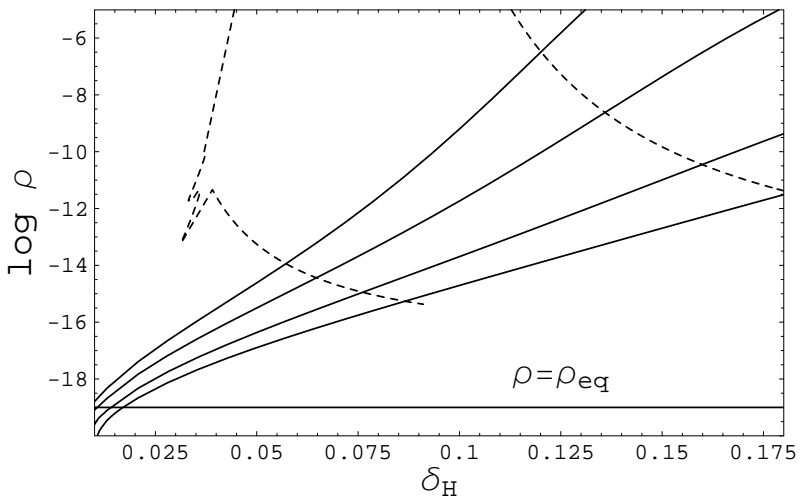

Figure 2. Density of the SDMC in dependence on the perturbation value $\delta_{H}$ for different clumps' masses $M=10^{-11}$, $10^{-6}, 10^{-1}, 10^{2} M_{\odot}$ (from up to down). The $\mathrm{PBH}$ restrictions is shown for the thresholds of the PBH's formation $\delta_{\mathrm{th}}=1 / 3$ and $\delta_{\mathrm{th}}=0.7$ (bottom and top dashed curves, respectively). The local minimum on the dashed curve corresponds to the Hawking evaporating PBHs.

The SDMCs are restricted by the process of the PBH formation. The mass of DM in some spherical volume is connected to the mass of the radiation at the moment of the horizon crossings as [7]

$$
M_{\mathrm{H}} \simeq 2 \times 10^{5}\left(\frac{M}{0.1 M_{\odot}}\right)^{2 / 3} M_{\odot}
$$

therefore the PBHs restrict the SDMCs from the different mass region. The PBHs are formed in the tail of the Gaussian perturbations distribution, but SDMC are formed from perturbations of the rms order. The PBHs and SDMC are connected not individually but through their common spectrum of perturbation. As it's clear from the Fig 2, the PBH restriction are very sensitive to the threshold $\delta_{\text {th }}$ of the PBH formation. According to different calculations this threshold is $\delta_{\text {th }}=1 / 3-0.7$, where $\delta_{\text {th }}=1 / 3$ corresponds to the simple analytic arguments of B.J. Carr, and the numerically revealed critical gravitational collapse gives even $\delta_{\mathrm{th}}=0.7$ [38] . There is only small room for the SDMCs in the case $\delta_{\text {th }}=1 / 3$.

The small-scale clumps from high peaks form earlier in comparison with the larger objects. Therefore the small clumps are not destructed in the hierarchical clustering.

For illustration we consider the three particular examples, which are presented in the Table I]

1. The clumps with minimum mass $M \simeq 10^{-6} M_{\odot}$ in the case of the standard spectrum of perturbation, normalized on the 7-year WMAP data (power index of the primordial spectrum $n_{p}=0.963 \pm 0.014$ ).

2 . The SDMC with the same mass $M \simeq 10^{-6} M_{\odot}$ but formed at the RD stage from the peak with $\delta_{H}=0.05$.

3. The limiting case of SDMC: clumps are formed at $t \sim t_{\text {eq }}$ from $\delta_{\text {eq }} \sim 1$ perturbations. For example, we consider here the mass $M \simeq 0.1 M_{\odot}$, which correspond to the DM mass-scale of the quark-gluon transition and to the mass of microlensing objects.

\section{B. Non-spherical model}

In general the shapes of the real perturbations are not spherical. To explore the importance of this nonsphericity one must go beyond the spherical approximation. We model the non-spherical evolution of SDMC by the homogeneous ellipsoid. Though this model doesn't take into account the internal structure of the real SDMC, it's useful for the calculation of the non-spherical outer layers evolution, where the most of mass is concentrated. For the detailed description of the nonlinear homogeneous ellipsoid model see [39]. We consider here the small departures from the spherical shape, it will allow us to simplify the full nonlinear problem, and this approximation is enough for our purposes.

The total potential of the perturbation

$$
\phi=\frac{1}{2} \Phi_{\alpha \beta}(t) r^{\alpha} r^{\beta}
$$

includes the potentials of ellipsoid (excess of the DM), homogeneous background and tidal forces:

$$
\Phi=\Phi_{\mathrm{el}}+\Phi_{\mathrm{bg}}+\Phi_{\mathrm{sh}}, \quad \Phi_{\mathrm{bg}}=4 \pi G \bar{\rho}(t) I / 3,
$$

where $I$ is the unit matrix. We neglect here the tidal term $\Phi_{s h}$ and a possible ellipsoid's rotation. Evolution is described by the equations

$$
\frac{d^{2} S^{\alpha \beta}}{d t^{2}}=-\Phi^{\alpha \gamma} S^{\gamma \beta}
$$

The matrix of the ellipsoid in the frame of it's main semiaxis can be written as

$$
S=\left\|\begin{array}{llll}
a & & \\
& b & \\
& & c
\end{array}\right\|=I r+\sigma, \Phi_{\mathrm{el}}=2 \pi G \rho_{e}\left\|\begin{array}{ccc}
A_{1} & & \\
& A_{2} & \\
& & A_{3}
\end{array}\right\|,
$$

where the density of ellipsoid $\rho_{e} \equiv M_{e} / V$ is given by

$$
\rho_{e}=\rho_{m}\left(\frac{1+\delta_{i}}{b^{3}}-1\right) .
$$

The coefficients of the potential are 39]

$$
A_{1}=a b c \int_{0}^{\infty} \frac{d \lambda}{\left(a^{2}+\lambda\right)\left[\left(a^{2}+\lambda\right)\left(b^{2}+\lambda\right)\left(c^{2}+\lambda\right)\right]^{1 / 2}},
$$

and $A_{2}, A_{3}$ can be written in the similar way. We denote $\Delta=\operatorname{Tr}(\sigma)$ and expand the potential up to the first power in the variables $\sigma \ll 1$ as

$$
\Phi_{\mathrm{el}}=2 \pi G \rho_{e}\left\{\frac{2}{3}\left(1+\frac{2}{5} \frac{\Delta}{r}\right) I-\frac{4}{5} \frac{\sigma}{r}\right\} .
$$

At the initial moment $t_{i}$ we choose the radius $r$ so that $\Delta\left(t_{i}\right)=0$, then it follows from (13) that $\Delta(t)=0$ at any time $t$ (similar truck was used e. g. in [40]). 
In the zero order $\sigma=0$ we have the same Eq. (6). In the next order we obtain the equation for the $\sigma$

$$
\frac{d^{2} \sigma}{d t^{2}}=\frac{4 \pi}{15} G \rho_{e} \sigma-\frac{4 \pi}{3} G\left(2 \rho_{r}+\rho_{m}\right) \sigma
$$

With the parametrization $\sigma=a(y) s(y) \xi$, the equation for the new function $s(y)$ can be written as

$$
y(y+1) s^{\prime \prime}+\left(1+\frac{3}{2} y\right) s^{\prime}-\frac{1}{10}\left(\frac{1}{b^{3}}-1\right) s=0 .
$$

Let us consider the initial conditions for the homogeneous ellipsoid at the RD-stage in the conformal Newtonian frame. At the scales $r \gg c t$ one has $\delta_{r}=-2 \Phi=$ const, $\delta_{i}=(3 / 4) \delta_{r, i}=-(3 / 2) \Phi_{i}$. The solution for the relativistic potential is 35 ]

$$
\Phi(\eta, \vec{k})=\Phi_{i}(\vec{k}) \frac{3 \pi^{1 / 2}}{2^{1 / 2}\left(u_{s} k \eta\right)^{3 / 2}} J_{3 / 2}\left(u_{s} k \eta\right),
$$

where $u_{s}=1 / \sqrt{3}$. The peculiar velocities $v_{j}$, which define the initial velocity of the ellipsoid's surface, are expressed as $v_{j}=\partial v / \partial x_{j}$, where the velocity potential 35]

$$
v(\vec{k})=-\frac{1}{\eta} \int_{0}^{\eta} d \eta^{\prime} \eta^{\prime} \Phi\left(\eta^{\prime}, \vec{k}\right)=-9 \Phi_{i}(\vec{k}) \frac{1}{\eta k^{2}} .
$$

The ellipsoidal top-hat perturbation has the form $\delta_{i}(\vec{x})=\delta_{i}=$ const if $(x / a)^{2}+(y / b)^{2}+(z / c)^{2} \leq 1$, and $\delta_{i}(\vec{x})=0$ otherwise. The Fourier transform of the ellipsoidal top-hat is [41]

$$
\delta_{i}(\vec{k})=\delta_{i}(2 \pi)^{3} a b c\left(\frac{\sin (\tilde{k})-\tilde{k} \cos (\tilde{k})}{2 \pi^{2} \tilde{k}}\right),
$$

where $\tilde{k}=\left[\left(a k_{x}\right)^{2}+\left(b k_{y}\right)^{2}+\left(c k_{z}\right)^{2}\right]^{1 / 2}$. Let us denote $\tilde{\vec{x}}=(x / a, y / b, z / c)$, then

$$
v(\tilde{\vec{x}})=\frac{1}{a b c} \int \frac{d^{3} \tilde{k}}{(2 \pi)^{3}} \frac{-9 \Phi_{i}(\vec{k}) e^{-i \tilde{\tilde{x}} \tilde{\vec{k}}}}{\eta\left[\left(\tilde{k}_{x} / a\right)^{2}+\left(\tilde{k}_{y} / b\right)^{2}+\left(\tilde{k}_{z} / c\right)^{2}\right]} .
$$

In the approximation of small nonspericity one can write

$$
v=v_{0}+\left.\frac{\partial v}{\partial a}\right|_{0} \Delta a+\left.\frac{\partial v}{\partial b}\right|_{0} \Delta b+\left.\frac{\partial v}{\partial c}\right|_{0} \Delta c+\cdots,
$$

where zero corresponds to the spherical zero-order case with $a=b=c$. The only nonzero derivative $v_{j}=\partial v / \partial x_{j}$ is over the correspondent axis $x_{j}$ for the linear terms in (24). After some algebra we have the initial conditions in the form

$$
\left.s\right|_{y_{i}}=s_{i} \quad \text { and }\left.\quad s^{\prime}\right|_{y_{i}}=\frac{3 \delta_{H} b_{i}^{3} s_{i}}{10 y_{i} \phi}
$$

for each component of $s$.

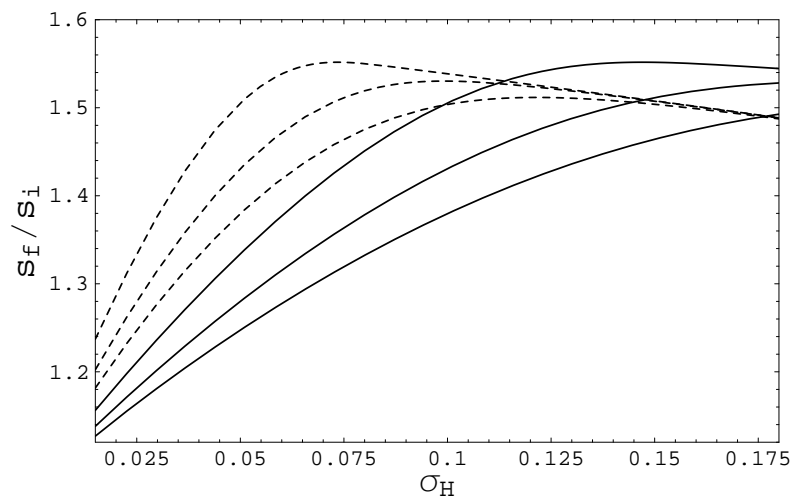

Figure 3. Asphericity growth $s_{f} / s_{i}$ in dependence on the r.m.s. perturbation value $\sigma_{H}$ at the horizon crossing for different clumps' masses $M=10^{-6}, 10^{-1}, 10^{2} M_{\odot}$ (from up to down). The solid and the dashed curves corresponds to the $\nu=1$ and $\nu=2$ peak hights, respectively, where $\nu \equiv \delta_{H} / \sigma_{H}$.

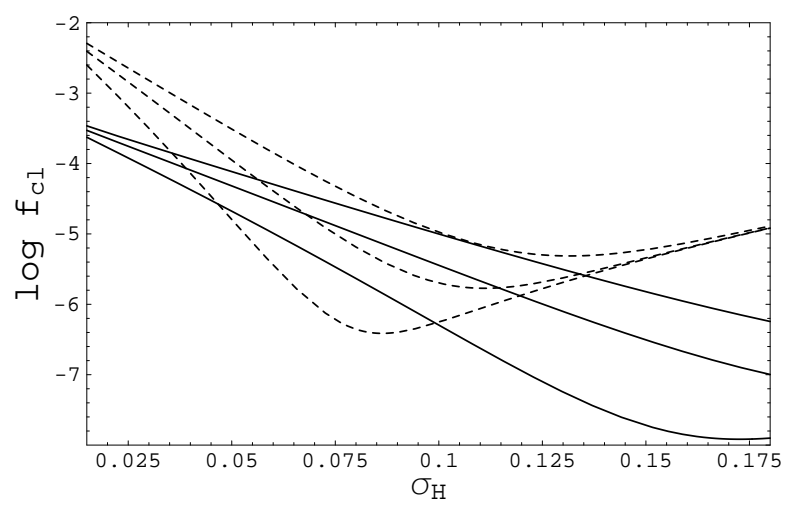

Figure 4. Fraction of clumps $f_{\mathrm{cl}}$ which formed by surviving the growth of anisotropy for clumps masses $M=10^{2}, 10^{-1}$, $10^{-6} M_{\odot}$ (from up to down). The solid and the dashed curves corresponds to the $\nu=1$ and $\nu=2$ peak highs, respectively.

We solve Eg. (19) numerically simultaneously with (6) for the peak highs $\nu=1$ and $\nu=2$, where $\nu$ is defined as $\nu \equiv \delta_{H} / \sigma_{H}$ and $\sigma_{H} \equiv\left\langle\delta_{H}^{2}\right\rangle^{1 / 2}$. The results are presented at the Fig. 3. We calculated the grow of the asphericity till the detachment of the object from the Hubble flow. Note that in the case of isocurvature perturbation the similar formalism (with initial $s^{\prime}\left(t_{i}\right)=0$ ) shows that the asphericity growth is small, $s$ changes by less then $10 \%$, therefore for the isocurvature perturbation the asphericity constraints are not strong. Now we return to the curvature perturbations. The boundary of the allowed asphericity is roughly $\Delta s_{f} / b_{f}<1$. From this condition the following criterion for the SDMC formation follows $\Delta s_{i} / b_{i}<\left(b_{f} / b_{i}\right)\left(\Delta s_{i} / \Delta s_{f}\right)$. The perturbations with larger $\Delta s_{i} / b_{i}$ will not form the clumps at the RDstage.

Now let us consider the distribution of the initial asphericity of perturbations. The distribution of Gaussian 


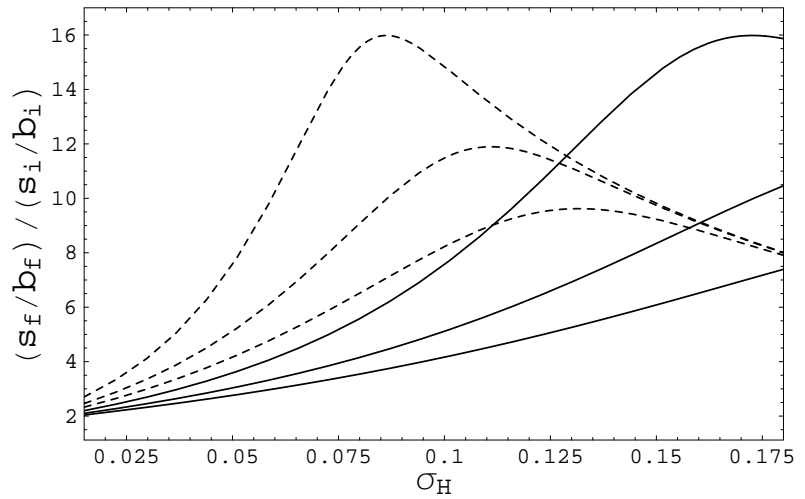

Figure 5. The same as at Fig. 3 but for the relative asphericity growth $\left(s_{f} / b_{f}\right) /\left(s_{i} / b_{i}\right)$.

perturbation over their shapes can be calculated with the help of results of [42], [43]. With the help of (17) the ellipticity of the potential of the ellipsoidal distribution can be expressed as

$$
e=\frac{\lambda_{1}-\lambda_{2}}{2 \sum \lambda_{i}} \simeq \frac{1}{5} \frac{\Delta s_{i}}{b_{i}}
$$

where $\lambda_{i}$ are the eigenvalues of the gravitational-shear tensor. The 2 nd variable prolateness is expressed as

$$
p=\frac{\lambda_{1}+\lambda_{3}-2 \lambda_{2}}{\sum \lambda_{i}}
$$

The distribution over eigenvalues $\lambda_{1} \geq \lambda_{2} \geq \lambda_{3}$ was found in [42] in the form

$$
\begin{aligned}
p\left(\lambda_{1}, \lambda_{2}, \lambda_{3}\right) & =\frac{15^{3}}{8 \pi \sqrt{5} \sigma^{6}} \exp \left(-\frac{3 I_{1}^{2}}{\sigma^{2}}+\frac{15 I_{2}}{2 \sigma^{2}}\right) \times \\
& \times\left(\lambda_{1}-\lambda_{2}\right)\left(\lambda_{2}-\lambda_{3}\right)\left(\lambda_{1}-\lambda_{3}\right),
\end{aligned}
$$

where $\sigma$ is the r.s.m. perturbation, $I_{1}=\lambda_{1}+\lambda_{2}+\lambda_{3}$, and $I_{2}=\lambda_{1} \lambda_{2}+\lambda_{2} \lambda_{3}+\lambda_{1} \lambda_{3}$. This distribution can be expressed through $e$ and $p$ variables as [44]

$$
g(e, p \mid \nu)=\frac{1125}{\sqrt{10 \pi}} e\left(e^{2}-p^{2}\right) \nu^{5} \mathrm{e}^{-\frac{5}{2} \nu^{2}\left(3 e^{2}+p^{2}\right)},
$$

where $\nu \equiv \delta / \sigma$. We use this distribution, integrated over $p$ in the range $-e<p<e$, for the calculations of the fraction of the formed SDMC. The results are shown at Fig. 4. Therefore the effect of asphericity can diminish the number of the formed SDMC by several orders.

The smallness of $f_{\mathrm{cl}}$ can also be explained by the following way. The absolute values of $s$ grows only moderately, and the fraction $s_{f} / s_{i}$ is not large. But the relative asphericity grows significantly because of the large value of $b_{i} / b_{f}$. The ratio of $s_{f} / b_{f}$ and $s_{i} / b_{i}$ is shown at Fig. 5. Therefore the initial asphericity $s_{i} / b_{i}$ should be very small for the final asphericity to be $s_{f} / b_{f}<1$. The probability distribution depends namely on the relative asphericity $s / b$. This is the reason why the initial distribution of asphericity (29) gives the huge suppression of the formed clumps fraction.

\section{SECONDARY ACCRETION AND THE FORMATION OF ULTRACOMPACT MINIHALOES}

\section{A. Smooth initial perturbation v.s. compact seed}

There are two distinct but complementary models of the clumps formation: evolution of the initially smooth perturbation, and the secondary accretion onto the previously formed seed. Let us consider the dust-like stage of the universe. The global parameters of the formed clump can be found from the simple top-hat model [45]. From the linear perturbation growth theory taking into account the gradual transition from the radiation to matter-dominated stage the expression follows [35]

$$
\delta(k, z) \simeq \frac{27}{2} \Phi_{i}(k) \frac{1+z_{\mathrm{eq}}}{1+z} \ln \left(0.2 k \eta_{\mathrm{eq}}\right),
$$

where $\Phi_{i}$ is the initial gravitational potential of the perturbation well outside the horizon. The formation time $t_{c}$ of the clump can be found from the relation $\delta\left(t_{c}\right)=\delta_{c}$, where $\delta_{c}=3(2 \pi)^{2 / 3} / 20 \approx 1.686$ (see e. g. 466]). The mean density of the clump is $\bar{\rho}_{\text {int }}=\varkappa \bar{\rho}\left(t_{c}\right)$, where $\varkappa=18 \pi^{2} \approx 178$, and its virial radius

$$
R=\left(\frac{3 M}{4 \pi \bar{\rho}_{\text {int }}}\right)^{1 / 3} .
$$

Further growth of the clump is due to virialization of the new spherical layers, if the perturbation extends to the larger scales till the regions, where $\delta=0-$ this is the boundary of the perturbation region with total energy $E<0$. The statistics of the clumps is determined by the power-spectrum of perturbations, normalized on the observational data. One can use in the (30) the spectrum of potential $\mathcal{P}_{\Phi}$ for which $A_{\Phi}=(4 / 9) A_{\mathcal{R}}$ in (1).

If these clumps were formed from the isolated density perturbations, they probably have the Gurevich-Zybin density profiles $\rho \propto r^{-1.8}$ [23], 24], 25] or near isothermal profile $\rho \propto r^{-2}$ [27]. The rather different profiles arise in the hierarchical clustering, if the clump was formed by the aggregation of smaller clumps. In this case the clump probably have the NFW or similar density profiles.

Let us discuss the secondary accretion scenario in more details. In the spirit of secondary accretion models, we assume that at the RD-stage there is the compact seed mass $M_{c}$, and the DM is distributed homogeneously around it. For $t \ll t_{\text {eq }}$ and for the mass $M \gg M_{c}$, the isocurvature perturbation of DM $\delta_{i}=M_{c} / M$ does not evolve. Indeed, according to the Meszarosh solution $\delta=\delta_{i}(1+3 x / 2)$ [45], where $x=a / a_{\text {eq }}$. This solution can easily be obtained from equation (6) in the linear approximation.

The secondary accretion begins at $t \sim t_{\mathrm{eq}}$. If we assume that the Hubble flow was not perturbed (such situation takes place for entropy perturbations), then one have to replace the $5 \delta / 3 \rightarrow \delta$ in the top-hat model [16], 
and the threshold for the object formation in this case is $\delta(t)=\tilde{\delta}_{c}=(3 \pi / 2)^{2 / 3} \approx 2.81$. Using the Meszarosh solution one finds the mass $M$ of the virialized object in dependence of the redshift

$$
\begin{aligned}
M(z) & =\frac{3}{2}\left(\frac{2}{3 \pi}\right)^{2 / 3} \frac{1+z_{\mathrm{eq}}}{1+z} M_{c} \\
& \approx 1.7 \times 10^{3}\left(\frac{M_{c}}{10^{2} M_{\odot}}\right)\left(\frac{1+z}{100}\right)^{-1} M_{\odot},
\end{aligned}
$$

where $(3 / 2)(2 /(3 \pi))^{2 / 3} \approx 0.53$. This numerical coefficient is about $1 / 2$ of the coefficient obtained in the work [9]. The virial radius of this clump is

$$
\begin{aligned}
r_{c} & =\frac{1}{3}\left(\frac{3}{4 \pi}\right)^{1 / 3} \frac{M^{4 / 3}}{\rho_{\mathrm{eq}}^{1 / 3} M_{c}} \\
& \simeq 3.2\left(\frac{M_{c}}{10^{2} M_{\odot}}\right)^{1 / 3}\left(\frac{100}{1+z}\right)^{4 / 3} \mathrm{pc} .
\end{aligned}
$$

Using the connection (33) between the halo mass and its radius, one finds the density profile of the "induced halo" at the distances where $M>M_{c}$ :

$$
\begin{aligned}
\rho(r) & =\left.\frac{1}{4 \pi r_{c}^{2}} \frac{d M\left(r_{c}\right)}{d r_{c}}\right|_{r_{c}=r} \\
& \simeq 3 \times 10^{-21}\left(\frac{r}{1 \mathrm{pc}}\right)^{-9 / 4}\left(\frac{M_{c}}{10^{2} M_{\odot}}\right)^{3 / 4} \mathrm{~g} \mathrm{~cm}^{-3} .
\end{aligned}
$$

In the case of non-compact central object, such as extended clusters of primordial black holes, the density profile does not match exactly the profile $\rho \propto r^{-9 / 4}$.

\section{B. Superdense clumps as the seeds}

The UCMHs can be formed around the existing SDMC. The structure of a UCMH around a SDMC, originated from the entropy perturbations, was considered in [6]. There is no principle difference with the SDMC originated from the peaks in the spectrum of the adiabatic perturbations. In both cases the rapid growth of UCMH begins only at $t \sim t_{\text {eq }}$.

In some sense a SDMC plays the role of the core radius of the UCMH. It's important that the secondary accretion density profile $\rho \propto r^{-9 / 4}$ definitely continues inward down to the radius of the SDMC. At the smaller radius the density profile of the UCMH is the SDMC's profile which may be the Gurevich-Zybin profile $\rho \propto r^{-1.8}$ with some core, although steeper profiles are also possible. In any case some mechanism may stop the density growth at the center of the SDMC (see Section [V]).

\section{Final mass of the ultracompact minihalos}

Total mass of induced halo increases with time as more and more remote areas around the seed are separated

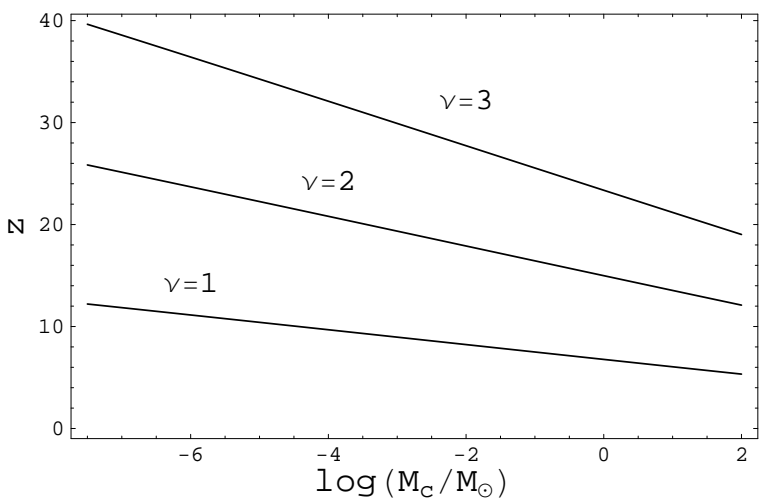

Figure 6. The red-shift $z$ of the growth termination according to Eq. 35 for $\nu=1,2$ and 3 fluctuations highs.

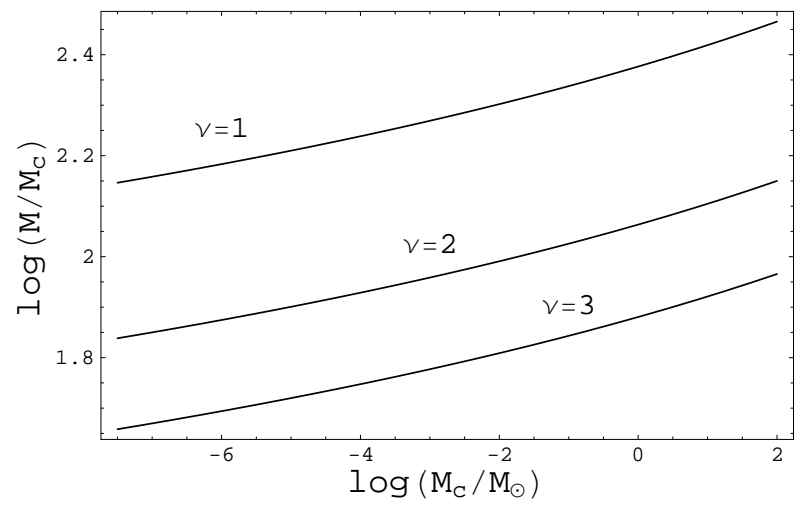

Figure 7. The final mass of the secondary accreted halo $M$ in relation to the seed mass $M_{c}$ for $\nu=1,2$ and 3 fluctuations highs.

from the cosmological expansion and virialized. The growth of the induced halo is terminated in an era of the nonlinear stage development of the normal inflationary perturbations of DM with mass which is of the order of the growing mass $M(t)$ of the induced halo at that epoch. The growth laws $\delta \propto t^{2 / 3}$ of the normal perturbations and the perturbations induced by the seed mass, are the same in the era of the matter dominance.

The particular UCMH is surrounded by several perturbations of the same mass-scale. At about half of them the perturbation is positive. Therefore, the condition of the growth ceasing from the side of positive perturbation is

$$
\nu \sigma_{\mathrm{eq}}(M)=\frac{9}{10} \frac{M_{c}}{M},
$$

where $\nu$ is the value of the density perturbation in terms of rms fluctuation $\sigma_{\text {eq }}(M)$. The right-hand side of equation (35) denotes the amplitude of the fluctuations caused by the mass $M_{c}$ with the factors discussed in the Section III A. The left side of the equation represents the usual Gaussian fluctuations at the time $t_{\text {eq }}$. Due to the 
large number of the surrounding perturbations the perturbations with the positive $\nu \sim 1$ will stop the UCMH growth from several directions and will destroy the regular secondary accretion. In addition, the negative perturbations $\nu<0$ will also dump the UCMH growth due to the lower density of DM inside them. Numerical solution of (35) gives the final average mass of the induced halo $M \sim\left(10^{1.5}-10^{2.5}\right) M_{c}$ in the range $M_{c} \sim 10^{-8}-10^{2} M_{\odot}$. The mechanism of the induced halo's growth stop, similar in some aspects to the one discussed in this section, was used in the works [17], 18], 19] for the calculation of the induced halo properties. The red-shift of the growth termination and the fraction $M / M_{c}$, found from the relations (35), $\delta\left(t_{c}\right)=\delta_{c}$ and known function $z(t)$, are shown at the Fig. 6, Fig. 7, respectively. The calculated redshift is roughly consistent with $z \sim 10-30$ predictions.

\section{MAXIMUM DENSITY IN THE CENTERS OF THE CLUMPS}

The density inside the clump grows towards the center. If the density stops growing at some radius $r=R_{c}$, then the region $r<R_{c}$ is referred to as "core". The evidences for the relative core radius $R_{c} / R \sim 10^{-2}$, where $R$ is the virial radius, are seen in the numerical simulations [1]. However, in another simulation [47] the core is not seen down to $R_{c} / R \sim 10^{-3}$; in this simulation the power-law growth continues down to the distance, where the numerical resolution fails. Let us discuss several possible restrictions on the maximum density in the clumps centres (core radius).

\section{A. Liouville's theorem restriction for the core radius}

The Liouville's theorem is known in the two equivalent formulations: the phase volume $\int d q d p$ is conserved or the distribution function in the phase space $f(p, q)=$ const. Therefore the probability density at the core (mean value of $f(p, q)$ over core volume) cannot exceed initial value due to Liouville's theorem. The only necessary condition for the theorem validity is the Hamiltonian character of the system. Unfortunately, $R_{c}$ can only be restricted, but not determined with the help of the Liouville's theorem applied only to the initial and final stages of clump evolution. This is because the entropy production in the intermediate processes [34]. By other words, the phase volume becomes dispersed during the evolution (see Fig. 8.3 in 35 ).

The distribution function can be estimated simply as $f_{c}=\rho_{c} / v^{3}$, where $\rho_{c}$ is the core density and $v$ is the characteristic velocity in the core. For the near isothermal density profile $\rho(r) \propto r^{-2}$ the $v$ is of the order of the virial velocity of the whole clump. We restrict the $R_{c}$ from the Liouville's theorem applied to the initial and final stages.
There are two sources of initial entropy or initial $\sigma$ : thermal velocities of DM particles at decoupling and peculiar velocities in the case of adiabatic density perturbations.

The thermal part can be attributed to the distribution function at the time of kinetic decoupling $t_{d}$ [35] (Section 8.3.2). The neutralino is nonrelativistic nondegenerate at $t_{d}$, therefore the good approximation for the distribution function at this moment is the Maxwell's distribution:

$$
f_{p}(p) d^{3} r d^{3} p=\frac{\rho_{m}}{m(2 \pi m k T)^{3 / 2}} e^{-\frac{p^{2}}{2 m k T}} d^{3} r d^{3} p,
$$

where $\rho_{m}$ is the density of DM, which is expressed through the temperature at any time by the using of the entropy conservation condition $g_{*} T^{3} a^{3}=$ const, where $g_{*}$ is the effective number of degrees of freedom at the temperature $T$, and $m$ is the mass of DM particle. The distribution function inside the core is less then the initial distribution function, which has the maximum value at $p=0$. Therefore we use the inequality $f_{c}<f_{p}(p=0)$. For the isothermal density profile in the clump this condition gives the restriction on the relative core radius

$$
\frac{R_{c}}{R}>\frac{2 \pi^{1 / 2} \bar{\rho}^{1 / 4} T_{d}^{3 / 4}}{3^{1 / 4} G^{3 / 4} M^{1 / 2} m^{3 / 4} \rho_{m}^{1 / 2}\left(t_{d}\right)} .
$$

The numerical examples for the case of $m=100 \mathrm{GeV}$ neutralinos with the temperature of the kinetic decoupling $T_{d} \simeq 25 \mathrm{MeV}$ are shown in the Table 1

Now we turn to the peculiar velocities, which are generated due to the gravitational instability and can play the role analogous to the thermal velocities in the $\mathrm{Li}$ ouville theorem restriction. Normally, the cut-off of the spectrum is not sharp. Even for the usual exponential cut off, e.g., the $2 k$ modes are present with sufficiently large amplitudes, where $k$ is the cut-off scale. The $2 k$ modes give the peculiar velocities which are distributed near isotropicaly, compared with the approximately radial $k$ modes. Therefore, the modes $k^{\prime}>k$ can be considered as approximately randomly directed in analogy with the thermal velocities in the Liouville theorem.

The peculiar velocity at some mass scale in the case of the flat metrics can be expressed as [45]

$$
\vec{v}=\frac{H a}{4 \pi} \nabla_{x} \int \frac{d^{3} x^{\prime} \delta\left(\vec{x}^{\prime}\right)}{\left|\vec{x}^{\prime}-\vec{x}\right|} .
$$

The growing mode comprises only $2 / 5$ fraction of (38). Just after the $t_{\mathrm{eq}}$ the falling mode ceased, the peculiar velocities developed and grow later as $\propto t^{1 / 3}$. We take the initial stage at $t=t_{\mathrm{eq}}$. The calculations are analogous to the thermal case with the replacement of the thermal velocity by the peculiar one. We obtain the minimum relative core radius in the form

$$
\frac{R_{c}}{R}=0.01 \delta_{\mathrm{eq}}^{9 / 2} \text {. }
$$

The numerical results of the calculations (37) and (39) are shown in the Table I. At the RD-stage the peculiar velocities are estimated according to the formalism presented in the Section 


\begin{tabular}{|c|c|c|c|c|c|}
\hline$M / M_{\odot}$ & $\bar{\rho}, \mathrm{g} \mathrm{cm}^{-3}$ & $\delta$ & $R_{c} / R$, Liouv. therm. & $R_{c} / R$, Liouv. pec. & $R_{c} / R$, annih. \\
\hline $10^{-6}$ & $3 \times 10^{-23}$ & $\delta_{\mathrm{eq}}=0.009$ & $4 \times 10^{-3}$ & $6 \times 10^{-12}$ & $2.6 \times 10^{-5}$ \\
\hline $10^{-6}$ & $4.2 \times 10^{-16}$ & $\delta_{H}=0.05$ & 0.24 & 0.1 & 0.1 \\
\hline 0.1 & $2.5 \times 10^{-17}$ & $\delta_{\mathrm{eq}} \simeq 1$ & $4 \times 10^{-4}$ & 0.01 & $2.5 \times 10^{-2}$ \\
\hline
\end{tabular}

Table I. The parameters of the clumps in the three examples and the relative core radius due to different effects.

\section{B. Annihilation criterion}

In the work [30] the maximum density in the center of the clump was estimated from the annihilation rate and the elapsed time:

$$
\rho\left(r_{\min }\right) \simeq \frac{m}{\langle\sigma v\rangle\left(t_{0}-t_{f}\right)}
$$

where $t_{0}$ is the current moment of time and $t_{f}$ is the formation moment of the clump. According to this estimate the core region becomes larger due to the loss of the particles at the orbits, that goes through the center of the clump. For the isothermal $\rho(r) \propto r^{-2}$ density profile, the corresponding relative core radius

$$
\frac{R_{c}}{R} \simeq\left(\frac{\langle\sigma v\rangle t_{0} \bar{\rho}}{3 m}\right)^{1 / 2}
$$

is presented in the Table I for the thermal production value $\langle\sigma v\rangle \simeq 3 \times 10^{-26} \mathrm{~cm}^{3} \mathrm{~s}^{-1}$ and $m=100 \mathrm{GeV}$. This approach assumes that the orbits passing through the center are not fulfilled after the particles annihilation.

The opposite case with the compensation of the particles loss was considered in [31] and [32]. In [31] it is found that the core radius is defined by the annihilation at the stage of the halo formation. The minimum radius was found from the condition that the annihilation time is of the order of the Jeans time, because this time defines the characteristic time of the density profile formation. This corresponds to the epoch of the halo formation. In the article 32] the core radius of the already formed clump was found in the assumption of the steady hydrodynamic bulk flow of the DM to the clump center. It was putted that the free fall time is equal to the annihilation time, and the center of the clumps is always fulfilled by the DM particles from the flow.

In the real clump there are mechanisms of the regeneration of the orbits with the small angular momentums, which goes through the center of the clump. These conditions result in the larger central density in comparison with (40). These orbits exist in some degree due to the clump's dynamical restructuring. Even the tidal forces from nearby stars and gravitational shocks at the galactic disk crossings lead to the changes of the clumps dynamical structure and appearance of the orbits with small angular momentum. The exact problem treatment requires the solution of the self-consistent kinetic equations for the clump structure with the influence of the external tidal forces.

\section{Possible mechanisms of the core formation}

In the above subsections the lower limits on the relative core radius were presented. It's clear from the Table I, that the Liouville restriction with the peculiar velocities can be the dominant effect in the formation of the core in the very dense clumps, formed just after the matterradiation equality.

Can other effects produce the core with the greater sizes? Dwarf and LSB galaxies tell us that some unknown effect is responsible for their large cores, which was not reproduced yet in the N-body simulations. First of all, the phase-space density is diluted by the entropy generation during the nonlinear hierarchical clustering [34]. In the work 34] this effect was explored for the galaxiessized halos, and 34 concluded that at the small scales the entropy generation is less effective, but the quantitative value is unclear.

Another affect is the tidal forces, which deflect particles, defocus them from the center of the clump at the stage of it's formation 33]. May be, similar effect define the break of the density profile in the clump [48]. Interesting analytic estimation of the core radius $R_{c} / R \simeq \delta_{e q}^{3}$ was obtained in [23], [24], [25] from the energy criterion and the falling mode influence. But the real mechanism of the core formation is still unclear.

\section{ANNIHILATION OF DM IN THE ULTRACOMPACT MINIHALOS}

Let us estimate the annihilation signal from the UCMHs seeded by the SDMCs. The annihilation rate in the single UCMH contains contributions from the three components, the SDMC $r<R$, the intermediate region $R \leq r \leq R_{e}$ and the outer halo $R_{e} \leq r \leq R_{h}$ formed by 
the secondary accretion at the dust-like stage,

$$
\dot{N}_{\mathrm{cl}}=4 \pi\left[\int_{0}^{R}+\int_{R}^{R_{e}}+\int_{R_{e}}^{R_{h}}\right] r^{2} d r \rho_{\mathrm{int}}^{2}(r) m^{-2}\left\langle\sigma_{\mathrm{ann}} v\right\rangle,
$$

where $\sigma_{\text {ann }}$ is the annihilation cross-section. The first integral in the square-brackets is equal to

$$
\dot{N}_{\mathrm{cl}}^{(1)}=\frac{3}{4 \pi} \frac{\left\langle\sigma_{\mathrm{ann}} v\right\rangle}{m^{2}} \frac{M^{2}}{R^{3}} S,
$$

where the function $S$ depends on the distribution of DM, and $S=1$ in the simplest case of the uniform density $\rho_{\text {int }}(r)=$ const. For the power-law density distribution $\rho \propto r^{-\beta}$ with relative core radius $x_{c}=R_{c} / R$ we have

$$
S\left(x_{c}, \beta\right)=\frac{(3-\beta)^{2}}{3(2 \beta-3)}\left(\frac{2 \beta}{3} x_{c}^{3-2 \beta}-1\right)\left(1-\frac{\beta}{3} x_{c}^{3-\beta}\right)^{-2} .
$$

In particular $S \simeq 4 /\left(9 x_{c}\right)$ for the profile $\rho \propto r^{-2}$ with $x_{c} \ll 1$. If $\beta \geq 1.5$ the contribution (43) is dominant in the (42), so one can neglect the two last integrals in (42). Note, however that in the case of UCMH with PBH seed the second integral would be dominant.

We parametrize the annihilation signal in the $\psi$ direction (with respect to Galactic center) over solid angle $\Delta \Omega$ by the usual way [49]:

$$
J_{\gamma}(E, \psi, \Delta \Omega)=9.4 \times 10^{-11} \frac{d F}{d E}\langle J(\psi)\rangle_{\Delta \Omega},
$$

where

$$
\frac{d F}{d E}=\left(\frac{100 \mathrm{GeV}}{m}\right)^{2} \sum_{F} \frac{\left\langle\sigma_{F} v\right\rangle}{10^{-26} \mathrm{sm}^{3} \mathrm{~s}^{-1}} \frac{d N_{\gamma}^{F}}{d E}
$$

and the astrophysical factor

$$
\langle J(\psi)\rangle_{\Delta \Omega}=\frac{1}{8.5 \mathrm{kpc}} \frac{1}{\Delta \Omega} \int d \Omega^{\prime} \int d L\left(\frac{\rho(r)}{0.3 \mathrm{GeV} \mathrm{sm}^{-3}}\right)^{2},
$$

where the integration goes along the line-of-sight. For the diffuse signal from the clumped DM

$$
\begin{aligned}
& \langle J(\psi)\rangle_{\Delta \Omega}= \\
& f_{\mathrm{cl}} S\left(\frac{\bar{\rho}_{\mathrm{int}}}{0.3 \mathrm{GeV} \mathrm{sm}^{-3}}\right) \int_{\text {l.o.s. }} \frac{d L}{8.5 \mathrm{kpc}}\left(\frac{\rho_{H}(r)}{0.3 \mathrm{GeV} \mathrm{sm}^{-3}}\right),
\end{aligned}
$$

where for the Galactic halo density profile $\rho_{H}(r)$ we use the NFW profile. For the assumed annihilational canal the gamma-rays are generated due to the pion productions and decays $\pi^{0} \rightarrow 2 \gamma$. Denote by $\eta_{\pi^{0}} \sim 10$ the number of photons per one decay.

The flux of gamma-rays from the annihilation may exceed the Fermi-LAT limits for some regions of the free parameters space. Here we consider the annihilation of the ordinary $m \sim 100 \mathrm{GeV}$ neutralino. There are several

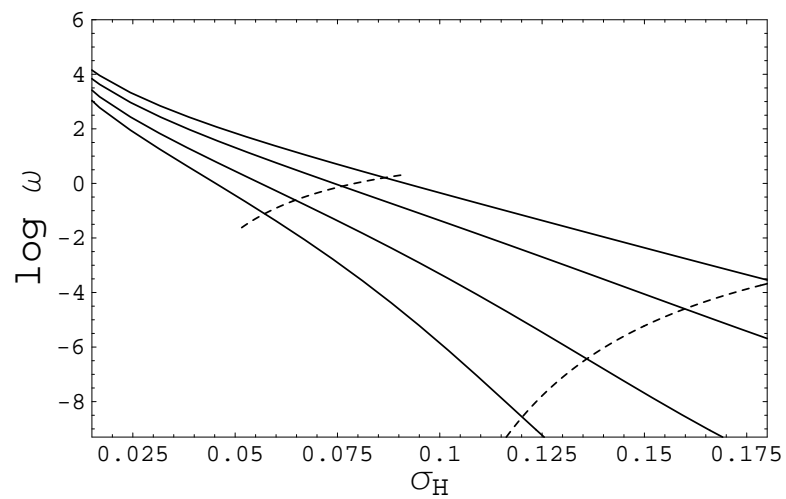

Figure 8. Solid lines show the upper limits on the parameter $\omega$ in the Eq. (49) for the clump masses $M=10^{2}, 10^{-1}, 10^{-6} M_{\odot}$ and $10^{-11} M_{\odot}$ (from up to down). The $\mathrm{PBH}$ restrictions with the thresholds $\delta_{\mathrm{th}}=1 / 3$ and $\delta_{\mathrm{th}}=0.7$ are shown by the left and right dashed curves, respectively.

uncertain parameters in the considered problem: core radius in the seed SDMC, mass $m$ and annihilation crosssection. We estimated the survived SHDM fraction $f_{\mathrm{cl}}$ in the Section IIB, but this value is very sensitive to the boundary of the allowed anisotropy $\Delta s_{f} / b_{f}<1$. Possibly, the boundary will be find in the future N-body simulation of the SDMCs formation. At the current level we still consider $f_{\mathrm{cl}}$ as the free parameter. Let us denote by the $\omega$ the following combination of parameters

$$
\omega=\left(\frac{\eta_{\pi^{0}}}{10}\right)\left(\frac{m}{100 \mathrm{GeV}}\right)\left(\frac{\langle\sigma v\rangle}{10^{-26} \mathrm{~cm}^{3} \mathrm{~s}^{-1}}\right)\left(\frac{f_{\mathrm{cl}}}{10^{-5}}\right) S .
$$

We compare the calculated annihilation signal in the Galactic anti-center direction with the Fermi-LAT diffuse extragalactic gamma-ray background $J_{\mathrm{obs}}(E>$ $\left.m_{\pi^{0}} / 2\right)=1.8 \times 10^{-5} \mathrm{~cm}^{-2} \mathrm{~s}^{-1} \mathrm{sr}^{-1}[50]$. It gives the conservative limit. The calculated upper limits on the parameter $\omega$ are shown at Fig. 8 . As one can see, the annihilation of ordinary neutralino in the SDMC is possible if one takes the effect of asphericity from Fig. (4 into account. Without this effect one would has $f_{\mathrm{cl}} \sim 1$, and the annihilation would exceed the Fermi-LAT limits even in the case of minimum possible annihilation cross-section which was found in [32].

\section{CONCLUSION}

In this paper we discussed several principle aspects of the superdense dark matter clumps (SDMC) and ultracompact minihalos (UCMH). In some particular cases we have calculated their properties. The range of the free parameters (first of all, describing unknown spectrum of perturbations at the small scales) are very broad, and it is hardly possible now to obtain the strict predictions. As a rule, only some restrictions are obtained from the annihilation or microlensings [51], 52] limits. The aim 
of this paper is to find the different types of DM clumps and their properties to avoid the mishmash, which are present occasionally in the literature about UCMH.

The DM clumps can originate at the RD-stage of the Universe evolution, as it was demonstrated first by [6] in the case of the entropy perturbations (see also some developments in [53] and [54]). High peaks (if they exist) atop the spectrum of the adiabatic perturbation can produce SDMC too [7]. During the SDMC evolution the asphericity of the perturbation can increase making the object highly non-spherical. It can prevent the SDMC formations. But the initial asphericity has a statistical distribution. In this paper we model the non-spherical objects as homogeneous ellipsoids in the approximation of a small asphericity. The calculated fraction of the formed clumps is in the range $f_{\mathrm{cl}} \sim 10^{-8}-10^{-2}$ depending on the value of perturbation at the horizon crossing $\delta_{H}$ and on mass-scale $M$, see Fig. 4 .

Virialisation at the RD stage proceeds similar to the virialization at the dust-stage if the clump detached from the cosmological expansion and its DM density exceeds locally the mean radiation density. The difference exists only for the very elongated fluctuations. Such fluctuations can be ripped apart and disintegrated without the SDMCs formation.

At the dust stage the secondary accretion can form UCMHs around the seed PBHs or SDMCs. The induced halos around these seeds grow until they begin to feel the surrounding density fluctuations. According to our calculations, the final mass of the UCMH exceeds the seed mass approximately by two orders.

The maximum density in the center of a clump may be restricted by some mechanisms. The Liouville theorem and annihilation criterion give only the upper limits on the central density, and these limits are particle-model dependent. In this work we calculated the Liouville theorem's limits for the thermal and peculiar velocities as the initial data. We have shown that the Liouville restriction can be the dominant effect for the formation of the core in those clumps, which originated just after the matter-radiation equality. In the case of SDMCs the annihilation criterion can also restrict the core radius. It's possible that there is some pure gravitational mechanism like tidal forces or entropy generation, which limits the central density and produces the central core. But such a mechanism is not reliably established.

The observational manifestation of the clumps is due to the annihilation of the DM particles inside them, see e. g. 55]. The particular calculations of the gamma-ray signal require the choice of some particular model and parameters for the clumps and DM particles. As we have shown, the initial asphericity diminishes the number of the formed SDMCs and lowers the anticipated annihilation signals from them. Even annihilation of ordinary $\sim 100 \mathrm{GeV}$ neutralinos in the SDMCs without exceeding of the Fermi-LAT observational limits is possible if one takes the effect of asphericity into account. By using the Fermi-LAT flux as the upper limit, we obtained the new restrictions on the combination of parameters for SDMCs and DM particles, see Fig. 8

\section{ACKNOWLEDGMENTS}

Authors thanks A.G. Doroshkevich and Referee for useful comments. The study was supported by the Ministry of education and science of Russia, project 8525; and in part by the grants OFN-17, and NSh-871.2012.2.
[1] J. Diemand, B. Moore and J. Stadel, Earth-mass darkmatter haloes as the first structures in the early Universe, Nature 433 (2005) 389.

[2] V. Berezinsky, V. Dokuchaev and Y. Eroshenko, Remnants of dark matter clumps, Phys. Rev. D 77 (2008) 083519 ; arXiv:0712.3499 [astro-ph].

[3] A.M. Green, S. Hofmann, D.J. Schwarz, The first WIMPy halos, JCAP $0508 \quad 003 \quad$ (2005); arXiv:astro-ph/0503387.

[4] T. Bringmann, "Particle models and the small-scale structure of dark matter", New J. Phys. 11105027 (2009); arXiv:0903.0189 [astro-ph.CO].

[5] V. Berezinsky et al., Superdense cosmological dark matter clumps, Phys. Rev. D 81 (2010) 103529; arXiv: $1002.3444 \mathrm{v} 2$ [astro-ph.CO].

[6] E. W. Kolb and I. I. Tkachev, Large Amplitude Isothermal Fluctuations And High Density Dark Matter Clumps, Phys. Rev. D 50 (1994) 769; arXiv:astro-ph/9403011

[7] V.I. Dokuchaev and Yu.N. Eroshenko, A common origin of neutralino stars and supermassive black holes, J. Exp. Theor. Phys. 94 (2002) 1; arXiv:astro-ph/0202021.
[8] P. Scott and S. Sivertsson, Gamma-Rays from Ultracompact Primordial Dark Matter Minihalos, Phys. Rev. Lett. 103 (2009) 211301; arXiv:0908.4082 v5 [astro-ph.CO].

[9] M. Ricotti, A. Gould, A New Probe of Dark Matter and High-Energy Universe Using Microlensing, Astrophys. J. 707(2009) 979; arXiv:0908.0735v2 [astro-ph.CO].

[10] A.S. Josan, A.M. Green, Gamma-rays from ultracompact minihalos: potential constraints on the primordial curvature perturbation, Phys. Rev. D 82 (2010) 083527; arXiv:1006.4970v2 [astro-ph.CO].

[11] Y. Yang et al., New Constraints on Primordial Minihalo Abundance Using Cosmic Microwave Background Observations, Phys. Rev. D 84 (2011) 043506; arXiv:1109.0156v3 [astro-ph.CO].

[12] Y. Yang et al., The Abundance of New Kind of Dark Matter Structures, Eur. Phys. J. Plus 126 (2011) 123; arXiv:1112.6228v1 [astro-ph.HE].

[13] Y. Yang et al., Constraints on ultracompact minihalos from extragalactic $\gamma$-ray background, JCAP 12 (2011) 020; arXiv:1112.6229v1 [astro-ph.CO].

[14] T. Bringmann, P. Scott, Y. Akrami, Improved constraints on the primordial power spectrum at small scales from ul- 
tracompact minihalos, arXiv:1110.2484 1 [astro-ph.CO].

[15] S. Shanderaet al., Number Counts and Non-Gaussianity, arXiv:1211.7361 [astro-ph.CO].

[16] E. Bertschinger, Self-similar secondary infall and accretion in an Einstein-de Sitter universe, Astrophys. J. Supp. 58 (1985) 39.

[17] V.I. Dokuchaev, Yu.N. Eroshenko, Stochastic Correlation Model of Galactic Bulge Velocity Dispersions and Central Black Holes Masses, Astron. Lett. 27 (2001) 759; arXiv:astro-ph/0202019v1.

[18] V.I. Dokuchaev, Yu.N. Eroshenko, Origin of Correlations between Central Black Holes Masses and Galactic Bulge Velocity Dispersions, Astron. Astrophys. Trans. 22 (2003) 727; arXiv:astro-ph/0209324v1.

[19] K.J. Mack, J.P. Ostriker and M. Ricotti, Growth of Structure Seeded by Primordial Black Holes, Astrophys. J. 665 (2007) 1277; arXiv:astro-ph/0608642 1.

[20] B.C. Lacki and J.F. Beacom, Primordial Black Holes as Dark Matter: Almost All or Almost Nothing, Astrophys. J. 720 (2010) L67L71; arXiv:1003.3466 [astro-ph.CO].

[21] D. Zhang, Impact of Primordial Ultracompact Minihaloes on the Intergalactic Medium and First Structure Formation, Mon. Not. Roy. Astron. Soc. 418 (2011) 1850; arXiv:1011.1935 [astro-ph.CO].

[22] R. Saito, S. Shirai, Primordial Black Hole as a Source of the Boost Factor, Phys. Lett. B 697 (2011) 95; arXiv:1009.1947v1 [hep-ph].

[23] A.V. Gurevich and K.P. Zybin, Nondissipative gravitational turbulence, Sov. Phys. JETP (1988) 671.

[24] A.V. Gurevich and K.P. Zybin, Nondissipative gravitational turbulence in the expanding Universe, Sov. Phys. JETP 67 (1988) 1957.

[25] A.V. Gurevich and K.P. Zybin, Large-scale structure of the Universe. Analytic theory., Sov. Phys. Usp. 165 (1995) 723 [Uspekhi Fizicheskikh Nauk, 165 (1995) 723].

[26] D. Anderhalden and J. Diemand, Density Profiles of CDM Microhalos and their Implications for Annihilation Boost Factors, Journal of Cosmology and Astroparticle Physics, 04 (2013) 009; arXiv:1302.0003 [astro-ph.CO].

[27] M. Vogelsberger et al., Caustics in growing Cold Dark Matter Haloes, Monthly Notices of the Royal Astronomical Society 400 (2009) 2174; arXiv:0906.4341 [astroph.CO].

[28] Y. Yang, G. Yang, and H. Zong, Dark Matter Decay and the Abundance of Ultracompact Minihalos, Europhys. Lett. 101 (2013) 69001; arXiv:1210.1409 [astro-ph.CO].

[29] Y. Yang, G. Yang and H. Zong, Neutrino signals from ultracompact minihalos and constraints on the primordial curvature perturbation, Phys. Rev. D 87 (2013) 103525; arXiv:1305.4213 [astro-ph.CO].

[30] P. Ullio et al., Cosmological dark matter annihilations into $\gamma$-rays: A closer look, Phys. Rev. D 66 (2002) 123502; arXiv:astro-ph/0207125 1 .

[31] V.S. Berezinsky, A.V. Gurevich and K.P. Zybin, Distribution of dark matter in the galaxy and the lower limits for the masses of supersymmetric particles, Phys. Lett. B 294 (1992) 221.

[32] V. Berezinsky, A. Bottino and G. Mignola, On neutralino stars as microlensing objects, Phys. Lett. B 391 (1997) 355; arXiv:astro-ph/9610060

[33] V. Berezinsky, V. Dokuchaev and Y. Eroshenko, Smallscale clumps in the galactic halo and dark matter annihilation, Phys. Rev. D 68 (2003) 103003; arXiv:astro-ph/0301551.
[34] A.G. Doroshkevich, V.N. Lukash, E.V. Mikheeva, A solution of the problems of cusps and rotation curves in dark matter halos in the cosmological standard model, Phys. Usp. 55 (2012) 3 [Uspekhi Fizicheskikh Nauk 182 (2012) $3]$.

[35] D.S. Gorbunov, V.A. Rubakov, Introduction to the Theory of the Early Universe Hot Big Bang Theory. Inflationary Theory. World Scientific Publishing Co Pte Ltd, 2011.

[36] D. Lynden-Bell, Statistical mechanics of violent relaxation in stellar systems, Monthly Notices of the Royal Astronomical Society, 136 (1967) 101.

[37] S.D.M. White, Violent Relaxation in Hierarchical Clustering, Proceedings of the 36th Herstmonceux Conference, "Gravitational Dynamics", editors O. Lahav, E. Terlevich, R. Terlevich; arXiv:astro-ph/9602021 v1.

[38] J.C. Niemeyer, K. Jedamzik, Dynamics of Primordial Black Hole Formation, Phys. Rev. D 59 (1999) 124013; arXiv:astro-ph/9901292 1 .

[39] D.J. Eisenstein, A. Loeb, An Analytical Model for the Triaxial Collapse of Cosmological Perturbations, Astrophys. J. 439 (1995) 520; arXiv:astro-ph/9405012 v1.

[40] N.A. Zabotin, P.D. Naselskii, A.G. Polnarev, HighAmplitude Peaks of Density Disturbances and the Formation of Primordial Black-Holes in the Dust like Universe, Soviet Astronomy, 31 (1987) 353.

[41] C.G. Koay, J.E. Sarlls, E. Özarslan, Three-dimensional analytical magnetic resonance imaging phantom in the Fourier domain, Magnetic Resonance in Medicine $\mathbf{5 8}$ (2007) 430.

[42] A.G. Doroshkevich, Spatial structure of perturbations and origin of galactic rotation in fluctuation theory, , Astrophysics, 6 (1970) 320.

[43] J.M. Bardeen, et al., The statistics of peaks of Gaussian random fields, Astrophysical Journal, 304 (1986) 15.

[44] R.K. Sheth, H.J. Mo, G. Tormen, Ellipsoidal collapse and an improved model for the number and spatial distribution of dark matter haloes, Mon. Not. Roy. Astron. Soc. 323 (2001) 1; arXiv:astro-ph/9907024.

[45] P.J.E. Peebles, Large-Scale Structure of the Universe, Princeton University Press, Princeton, USA, 1993.

[46] C. Lacey, S. Cole, Merger rates in hierarchical models of galaxy formation, Mon. Not. R. Astron. Soc. 262 (1993) 627.

[47] T. Ishiyama, J. Makino, T. Ebisuzaki, Gammaray Signal from Earth-mass Dark Matter Microhalos, The Astrophysical Journal Letters, 723 (2010) L195; arXiv:1006.3392 2 [astro-ph.CO].

[48] P. Sikivie, I.I. Tkachev and Y. Wang, The secondary infall model of galactic halo formation and the spectrum of cold dark matter particles on Earth, Phys. Rev. D 56 (1997) 1863; arXiv:astro-ph/9609022 1.

[49] L. Bergstrom et al., Clumpy neutralino dark matter, Phys. Rev. D 59 (1999) 043506.

[50] A.A. Abdo et al., The Spectrum of the Isotropic Diffuse Gamma-Ray Emission Derived From First-Year Fermi Large Area Telescope Data, Phys. Rev. Lett. 104 (2010) 101101; arXiv:1002.3603v1 [astro-ph.HE].

[51] F. Li, A.L. Erickcek, N.M. Law, A new probe of the smallscale primordial power spectrum: astrometric microlensing by ultracompact minihalos, arXiv:1202.1284 1 [astroph.CO]. 
[52] E. Zackrisson, et al, Hunting for dark halo substructure using submilliarcsecond-scale observations of macrolensed radio jets, Mon. Not. Roy. Astron. Soc. 431 (2013) 2172, arXiv:1208.5482 [astro-ph.CO].

[53] M.Yu. Khlopov, A.S. Sakharov, D.D. Sokoloff, The nonlinear modulation of the density distribution in standard axionic CDM and its cosmological impact, Nuclear Physics B - Proceedings Supplements 72 (1999) 105;
arXiv:hep-ph/9812286

[54] M. Khlopov, Primordial Nonlinear Structures and Massive Black Holes from Early Universe, Journal of Physics: Conference Series 66 (2007) 012032.

[55] K.M. Belotsky, A.A. Kirillov, M.Yu. Khlopov, Gammaray evidences of the dark matter clumps, arXiv:1212.6087 [astro-ph.HE]. 